\title{
Non-osteogenic muscle hypertrophy in children with McArdle disease
}

\author{
I. Rodríguez-Gómez ${ }^{1,2} \cdot$ A. Santalla 2,3 $\cdot$ J. Díez-Bermejo $^{4}$ - D. Munguía-lzquierdo ${ }^{2,3} \cdot$ L. M. Alegre ${ }^{1,2}$. \\ G. Nogales-Gadea ${ }^{5,6} \cdot$ J. Arenas $^{4,6} \cdot$ M. A. Martín ${ }^{4,6} \cdot$ A. Lucía ${ }^{2,7} \cdot$ I. Ara ${ }^{1,2}$ (D)
}

Received: 24 January 2018 / Revised: 28 February 2018 / Accepted: 5 March 2018

(C) SSIEM 2018

\begin{abstract}
Introduction McArdle disease is an inborn disorder of muscle glycogen metabolism that produces exercise intolerance, and has been recently associated with low values of lean mass (LM) and bone mineral content (BMC) and density (BMD) in affected adults. Here we aimed to study whether this bone health problem begins in childhood.

Methods Forty children and adolescents were evaluated: 10 McArdle disease and 30 control children (mean age of both groups, $13 \pm 2 \mathrm{y}$ ). Body composition was evaluated by dual-energy X-ray absorptiometry and creatine kinase (CK) levels were determined in the patients as an estimate of muscle damage.

Results Legs bone mass was significantly lower in patients than in controls ( $-36 \%$ for BMC and $-22 \%$ for BMD). Moreover, patients had significantly higher LM values in the legs than controls, whereas no difference was found for fat mass. CK levels were positively associated with LM in McArdle patients. A correlation was found between LM and BMD variables in the control group but not in McArdle patients.

Conclusion We have identified a 'non-osteogenic muscle hypertrophy' in children with McArdle disease. This phenomenon warrants special attention since low osteogenesis at an early age predicts a high risk for osteoporosis later in life.
\end{abstract}

Communicated by: Bridget Wilcken

Electronic supplementary material The online version of this article (https://doi.org/10.1007/s10545-018-0170-7) contains supplementary material, which is available to authorized users.

I. Ara

ignacio.ara@uclm.es

1 GENUD Toledo Research Group, Universidad de Castilla-La Mancha, Avda Carlos III s/n, 45071 Toledo, Spain

2 CIBER of Frailty and Healthy Aging (CIBERFES), Madrid, Spain

3 Department of Sport and Computer Science, Section of Physical Education and Sports, Faculty of Sport, Universidad Pablo de Olavide, Sevilla, Spain

4 Research Institute Hospital 12 de Octubre, Madrid, Spain

5 Neuromuscular and Neuropediatric Research Group, Institut d'Investigació en Ciències de la Salut Germans Trias i Pujol, Campus Can Ruti, Universitat Autònoma de Barcelona, Badalona, Spain

6 CIBER Rare Disorders (CIBERER), Madrid, Spain

7 School of Research and Doctorate Studies, Universidad Europea de Madrid, Madrid, Spain

\section{Introduction}

McArdle disease (glycogen storage disease (or 'glycogenosis') type $\mathrm{V}$, myophosphorylase deficiency) is an inborn disorder of skeletal-muscle carbohydrate metabolism characterized by failure of muscle glycogen breakdown (Santalla et al. 2014a, b). Recent findings from the Spanish registry of McArdle patients (Santalla et al. 2017) show that the recognition of this disorder is increasing: 94 new patients (28\% of total) have been diagnosed since the first report in January 2011 (Lucia et al. 2012). The majority of patients report symptoms or medical problems during the first decade of life, but are not usually correctly diagnosed until adulthood (Santalla et al. 2017). The occurrence of frequent, long delays in genetic diagnosis would suggest that many patients remain undiagnosed or are misdiagnosed (Santalla et al. 2017). United Kingdom centres have reported the same issue (Scalco et al. 2017).

Given the above, it is important to comprehensively describe the clinical phenotype of McArdle disease in children. This is especially relevant when considering that, until a curative treatment is eventually available, current epidemiological data support the early adoption of an active lifestyle as the best option for these patients, which can attenuate the deleterious 
consequences of the disease, notably fixed muscle weakness (Nogales-Gadea et al. 2016). We recently reported that physically inactive adult McArdle patients had lower lean mass (LM) values in whole-body and regional sites than their healthy age- and gender-matched peers, as measured by dualenergy X-ray absorptiometry (DXA) (Rodríguez-Gómez et al. 2018). Importantly, bone mineral content (BMC) and density (BMD) were also lower in patients than in controls, and as such we identified poor bone health as a previously undescribed condition in McArdle patients.

Early infancy is a critical period for skeletal development, during which enhancing bone accretion can help to reduce the risk of osteoporosis and bone fractures later in life (Golden and Abrams 2014; Mitchell et al. 2015). It was therefore the aim of our study to compare the body composition (including $\mathrm{LM}$ and $\mathrm{BMC} / \mathrm{BMD}$ ) of McArdle patients aged $\leq 16$ years to that of healthy aged and gender-matched controls.

\section{Materials and methods}

\section{Subjects and procedure}

We followed a case-control, cross-sectional design. Patients (cases) were recruited for this study if they met the following criteria: (i) genetic diagnosis of McArdle disease, that is, identification of the two mutant alleles in the gene (PYGM) encoding myophosphorylase (Santalla et al. 2017); (ii) age $\leq$ 16 years; and (iii) having no condition contraindicating DXA (e.g., upcoming contrast tests). In total, 10 children with McArdle disease, five boys and five girls (13.8 \pm 2.2 years), who met all the inclusion criteria were included in the study. Data were collected from 06/2015 until 06/2017. The parents/ tutors of age- and gender-matched healthy children (controls) with DXA data previously collected in our laboratory (see below) were contacted again and consented to have their children's data used as historical controls for the present study. A total of 30 children, 15 boys and 15 girls (13.6 \pm 2.2 years), were recruited to meet a patient-control ratio of 1:3 from 2012 until 2017. During the same week of DXA assessment, anthropometric data (patients and controls) and serum creatine kinase (CK) levels (patients) were also assessed.

The study protocol was approved by the ethics committee of the Research Institute of the Hospital 12 de Octubre (Madrid, Spain; reference \# 16/081) and adhered to the tenets of the Declaration of Helsinki 1961 (revision Edinburgh 2008). Signed informed consent was obtained from all participants, who were informed about the aims and procedures of the study, as well as of the possible risks and benefits.

Genetic diagnosis Mutant PYGM alleles were identified in patients' muscle or blood samples as described elsewhere
(Santalla et al. 2017). All the pathogenic PYGM mutations identified in our study patients are shown in the Supplementary file.

\section{Anthropometry}

Height and body mass were obtained for each subject using a calibrated balance and stadiometer (Seca 711, Hamburg, Germany), immediately before DXA assessment. Both measurements were performed in the upright position, and children wore only their underwear. Height was recorded in the Frankfort plane with a precision of $1 \mathrm{~mm}$, and body mass was determined with $100 \mathrm{~g}$ precision. Body mass index (BMI) was calculated as body mass $(\mathrm{kg})$ divided by height $(\mathrm{m})$ squared $\left(\mathrm{kg} \cdot \mathrm{m}^{-2}\right)$.

\section{Body composition: lean, fat, and bone mass}

Body composition and bone mass in whole-body and regional sites were assessed in all the study participants using the same DXA instrument (Hologic QDR Discovery, Bedford, MA), in the Laboratory of the GENUD Toledo Research Group of the University of Castilla-La Mancha (Toledo, Spain). The DXA instrument was calibrated daily against a lumbar spine phantom following the manufacturer's guidelines. Physician's Viewer, APEX System Software Version 3.1.2. (Bedford, MA) was used to analyze all DXA scans. Scans were made with subjects in the supine position, wearing light clothing with no metal and no shoes or jewelry. LM (kg), fat mass (FM, $\mathrm{kg}$ ), BMC ( $\mathrm{g}$ ), and BMD (in $\mathrm{g} \cdot \mathrm{cm}^{-2}$ ) were calculated from total and regional analysis of the whole-body scans. To determine the composition of the arms, legs, pelvis and trunk regions, whole-body scans were submitted to a regional analysis; in addition, lower-leg composition was determined by a specific sub-regional analysis. BMC and BMD were also reported for the proximal region of the femur (total hip, greater trochanter, inter-trochanter, Ward's triangle and femoral neck). Full methodology has been described previously (RodríguezGómez et al. 2018). The pediatric bone Z-scores were calculated in each participant for the whole-body and femoral neck.

\section{Statistical analysis}

Statistical analyses were performed with the IBM SPSS statistics package version 24 (SPSS, Inc., Chicago, IL). The Kolmogorov-Smirnov test and graphical methods (normal probability plots) were used to determine the normal distribution of the variables. The characteristics of the study groups were determined through basic descriptive tests. Differences in body composition variables between patients and controls were defined by Student's $t$ test and analysis of covariance (ANCOVA). Age, mass, height, age of symptom onset, and frequency of rhabdomyolyisis episodes (as assessed by the number of reported episodes of myoglobinuria or 'dark 
urines') were entered as covariables in the analyses, with Bonferroni post hoc tests in the case of ANCOVA analysis. Effect sizes were reported with Hedge's $g$ test for comparisons of unequal sample size. Additionally, partial correlations were applied to identify potential associations between LM, bone mass and CK in the patients. The level of statistical significance was set at $p \leq 0.05$. Unless otherwise stated, results are reported as mean \pm standard error of measurement (SEM).

\section{Results}

All the patients had been previously described with pathogenic mutations that are known to cause McArdle disease in both alleles of the PYGM gene (Supplementary file, PYGM mutations identified in the study patients). Anthropometric and descriptive data for the McArdle and control groups are shown in Table 1. The group of McArdle patients represent $26 \%$ of the total Spanish patient population of the same age ( $\leq 16$ years) according to the recent update of patients (Santalla et al. 2017). Both groups were matched by age, body mass, height, and BMI.

\section{Body composition}

Between-group comparisons of total/regional LM and FM values, after controlling for the effect of age, body mass, height, age of symptoms onset, and frequency of rhabdomyolysis episodes, are shown in Table 2. LM in the lower extremities was significantly higher in patients than in controls ( $p<0.05$; Hedge's $\mathrm{g}=1.03$ and $0.39 ; 9.9 \%$ and $28.6 \%$ of difference, legs and lower legs, respectively). There were no significant between-group differences in FM at whole-body and regional sites or in \% body fat. Thus, the influence of body fat was similar for bone mass or LM, and this variable was excluded from subsequent analyses.

Table 3 shows between-group comparisons of BMC and BMD after controlling for the effect of age, body mass, height, age of symptoms onset and frequency of rhabdomyolysis episodes. BMD in the lower legs was significantly lower in McArdle patients than in controls $(p<0.01$; Hedge's $\mathrm{g}=$ $1.26 ; 22 \%$ of difference). Whole-body and legs' BMD

Table 1 Anthropometric and descriptive data

\begin{tabular}{lll}
\hline Variables & McArdle $(n=10)$ & Control $(n=30)$ \\
\hline Age (years) & $13.8 \pm 2.2$ & $13.6 \pm 2.2$ \\
Body mass $(\mathrm{kg})$ & $47.9 \pm 11.6$ & $56.8 \pm 17.4$ \\
Height $(\mathrm{cm})$ & $159.4 \pm 11.4$ & $162.1 \pm 11.4$ \\
BMI $\left(\mathrm{kg} / \mathrm{m}^{2}\right)$ & $18.6 \pm 2.6$ & $21.2 \pm 4.2$ \\
$\mathrm{CK}(\mathrm{U} / \mathrm{L})$ & $3902 \pm 3096$ & \\
\hline
\end{tabular}

Data are mean $\pm \mathrm{SD}$. BMI body mass index, $C K$ creatine kinase showed near-significant differences, with small effect sizes (Hedge's $\mathrm{g}=0.39$ in both cases; and 6.5 and $8.1 \%, p=0.09$ and $p=0.07$, respectively). Medium effect size of the differences was found for the femoral neck ( $p>0.05$; Hedge's $g=$ 0.52 and $7.7 \%$ of difference) and small effect sizes were found for the pelvis, arms, trochanter, and Ward's triangle (Hedge's g from 0.30 to 0.44 , from 3.2 to $10.1 \%$ ). When comparing BMC, significant differences were found only in the lower legs ( $p<0.01$; Hedge's $g=1.18 ; 35.8 \%$ of difference).

\section{Association between whole-body and legs lean mass and bone mass and CK}

The correlation coefficients between LM, BMD variables and CK are shown in Table 4. The main result was that CK was positively associated with whole-body and legs' LM ( $p \leq$ 0.01 ) in patients, but no significant associations were observed between LM and BMD. This latter finding was in contrast to that found in the control group.

\section{Discussion}

This study examined the differences in body composition between a representative sample of children with McArdle disease and age- and sex-matched controls. Our main, novel finding was that patients have significantly lower bone mass than their respective controls. By contrast, LM was similar between groups and was in fact higher in patients' lower limbs.

Childhood and adolescence are crucial periods for the development of the skeleton. It is therefore important to achieve a high peak bone mass during these stages in order to prevent osteoporotic fractures later in life (Rizzoli et al. 2010). In this regard, the idea that 'osteoporosis is a pediatric disease' is increasingly accepted (Bachrach 2014; Marrani et al. 2017) and, in fact, prevention from childhood is the most powerful strategy against non-communicable diseases such as osteoporosis (Vicente-Rodríguez 2006). In the present study, BMC values of the McArdle children were, on average 6\% lower and BMD values were, on average, $7 \%$ lower than those of their age- and gender-matched healthy peers. Moreover, the proportion of individuals with whole-body and femoral neck $\mathrm{Z}$-scores indicative of osteoporosis and risk of bone fracture level was greater in the McArdle patient group than in the control group (i.e. $75 \%$ of the patients assessed here showed lower-than-normal bone mass values). Similar findings on bone mass have been recently reported by us in adult McArdle patients, who have compromised bone health (Rodríguez-Gómez et al. 2018), and in patients with other myopathies, such as glycogenosis type I (Schonau et al. 2002), Ia (children/adolescents) (Rake et al. 2003) and III (Melis et al. 2016; Rake et al. 2003; Schwahn et al. 2002), or Pompe disease (Bertoldo et al. 2015). 
Table 2 Lean and fat mass composition from the total and regional body scans by group

\begin{tabular}{llllll}
\hline & \multicolumn{2}{l}{ Lean mass $(\mathrm{kg})$} & & \multicolumn{2}{l}{ Fat mass $(\mathrm{kg})$} \\
\cline { 5 - 6 } & McArdle $(\mathrm{n}=10)$ & Control $(n=30)$ & & McArdle $(\mathrm{n}=10)$ & Control $(\mathrm{n}=30)$ \\
\hline Whole body & $40.3 \pm 1.3$ & $38.0 \pm 0.7$ & & $13.2 \pm 1.3$ & $13.9 \pm 0.7$ \\
Trunk & $19.0 \pm 0.8$ & $18.5 \pm 0.4$ & & $5.3 \pm 0.8$ & $5.7 \pm 0.4$ \\
Arms (mean) & $2.0 \pm 0.1$ & $1.9 \pm 0.1$ & & $0.7 \pm 0.1$ & $0.7 \pm 0.4$ \\
Legs (mean) & $7.1 \pm 0.3$ & $6.4 \pm 0.1^{*}$ & & $2.9 \pm 0.3$ & $2.9 \pm 0.1$ \\
Lower legs (mean) & $0.7 \pm 0.1$ & $0.5 \pm 0.1^{*}$ & & $0.8 \pm 0.1$ & $1.0 \pm 0.1$ \\
\hline
\end{tabular}

Data are mean \pm SEM. $* p<0.05$ for McArdle vs. control group (comparison results adjusted by age, body weight, height, age of symptom onset and frequency of rhabdomyolysis episodes)
The finding of low BMD among children with McArdle disease despite their normal-to-high LM values might appear counterintuitive in light of the fact that high LM is probably the most important predictor for bone mass accrual during prepubertal growth in the overall population (Vicente-Rodríguez 2006). Such a muscle-bone relationship is presumably explained by the mechanostat theory, which posits that bone strength is regulated by modeling and remodeling processes that are dependent on the forces acting on the bones (Rauch et al. 2004; Schoenau and Frost 2002), with larger muscles usually exerting higher tensile forces on the bones they are attached to. Further, the result found here (high LM but low bone mass) appears to be specific to children and adolescents with McArdle disease, with adult patients showing rather low LM (unless they are active) as well as the 'normal', expected positive relation between whole-body LM and whole-body bone mass (Rodríguez-Gómez et al. 2018). As LM and BMD variables have not been previously reported together in patients with other types of glycogenosis, we cannot infer whether the findings reported here are specific to children with McArdle disease (glycogenosis type V) only.

Our findings suggest the existence of 'non-osteogenic muscle hypertrophy', in children and adolescents with McArdle disease. However, in the case of McArdle children, muscle hypertrophy is likely not associated with frequent exercise practice. Indeed, these children tend to refrain from practising sports activities that are typical for their age, especially in physical education classes or in the school playground (e.g., running, jumping) because these activities are the main trigger for symptom occurrence (Santalla et al. 2017). Yet, mechanical tensions induced by muscle contractions (i.e., the mechanostat theory) during such types of exercise are necessary to stimulate bone accretion (Eckhard Schoenau 2005). In children with McArdle disease, muscle hypertrophy could be the consequence, at least in part, of the ongoing muscle damage over life that characterizes this condition, as reflected by the high serum CK levels (a muscle damage marker) in our
Table 3 Bone mineral content (BMC) and density (BMD) from the whole-body and femoral regions

\begin{tabular}{|c|c|c|c|c|}
\hline & \multicolumn{2}{|l|}{$\mathrm{BMC}(\mathrm{g})$} & \multicolumn{2}{|l|}{$\operatorname{BMD}\left(\mathrm{g} \cdot \mathrm{cm}^{-2}\right)$} \\
\hline & McArdle $(\mathrm{n}=10)$ & Control $(n=30)$ & McArdle $(n=10)$ & Control $(n=30)$ \\
\hline \multicolumn{5}{|l|}{ Whole scan } \\
\hline Whole body & $1679.2 \pm 87.0$ & $1782.4 \pm 48.4$ & $0.93 \pm 0.03$ & $0.99 \pm 0.03^{0.09}$ \\
\hline Head & $389.0 \pm 19.2$ & $387.3 \pm 10.7$ & $1.64 \pm 0.07$ & $1.68 \pm 0.07$ \\
\hline Pelvis & $188.8 \pm 23.3$ & $225.7 \pm 12.9$ & $0.95 \pm 0.04$ & $1.03 \pm 0.04$ \\
\hline Arms (mean) & $102.0 \pm 6.1$ & $107.8 \pm 3.4$ & $0.63 \pm 0.02$ & $0.65 \pm 0.01$ \\
\hline Legs (mean) & $316.6 \pm 16.9$ & $346.3 \pm 9.4$ & $0.99 \pm 0.04$ & $1.07 \pm 0.04^{0.07}$ \\
\hline Lower legs (mean) & $124.8 \pm 18.8$ & $194.3 \pm 10.4 * *$ & $0.78 \pm 0.06$ & $1.00 \pm 0.03 * *$ \\
\hline \multicolumn{5}{|l|}{ Femoral regions } \\
\hline Proximal femur (mean) & $30.46 \pm 2.03$ & $29.3 \pm 1.4$ & $0.86 \pm 0.04$ & $0.89 \pm 0.03$ \\
\hline Femoral neck & $4.1 \pm 0.3$ & $3.5 \pm 0.2$ & $0.72 \pm 0.04$ & $0.78 \pm 0.02$ \\
\hline Trochanter & $5.3 \pm 0.5$ & $6.1 \pm 0.3$ & $0.64 \pm 0.05$ & $0.69 \pm 0.03$ \\
\hline Intertrochanteric zone & $21.2 \pm 1.6$ & $19.6 \pm 1.1$ & $0.96 \pm 0.04$ & $0.99 \pm 0.03$ \\
\hline Ward's triangle & $0.8 \pm 0.1$ & $0.9 \pm 0.0$ & $0.69 \pm 0.04$ & $0.76 \pm 0.03$ \\
\hline
\end{tabular}

Data are mean \pm SEM. $* * p<0.01$ for McArdle vs. control group (comparison results adjusted by age, body weight, height, age of symptom onset, and frequency of rhabdomyolysis episodes) 
Table 4 Partial correlation coefficients between $\mathrm{CK}$ and whole-body lean mass, legs lean mass, whole-body bone mineral density, and legs bone mineral density (all corrected for age, body weight, and height)

\begin{tabular}{|c|c|c|c|c|c|}
\hline & $\mathrm{CK}$ & Whole body LM & Legs LM & Whole body BMD & Legs BMD \\
\hline \multicolumn{6}{|l|}{ McArdle $(n=10)$} \\
\hline CK & - & $0.897 *$ & $0.912 * *$ & 0.098 & 0.341 \\
\hline Whole body LM & - & - & $0.909 * *$ & 0.450 & 0.587 \\
\hline Legs LM & - & - & - & 0.185 & 0.683 \\
\hline Whole body BMD & - & - & - & - & 0.477 \\
\hline Legs BMD & - & - & - & - & - \\
\hline \multicolumn{6}{|l|}{ Control $(\mathrm{n}=30)$} \\
\hline Whole body LM & - & - & $0.779 * *$ & $0.679 * *$ & $0.768 * *$ \\
\hline Legs LM & - & - & - & 0.354 & $0.417 *$ \\
\hline Whole body BMD & - & - & - & - & $0.930 * *$ \\
\hline Legs BMD & - & - & - & - & - \\
\hline
\end{tabular}

patients (i.e., mean levels of $\sim 4000 \mathrm{U} / \mathrm{L}$, or 20 -fold higher than the usual upper limits), which are in agreement with previous studies in children/adolescents with McArdle disease (GarciaBenitez et al. 2013; Perez et al. 2007).

Muscle damage is a major stimulus for muscle hypertrophy, with the subsequent skeletal muscle tissue inflammation and increased protein turnover being necessary for long-term hypertrophic adaptations to occur (Evans and Cannon 1991; Komulainen et al. 2000; Wernig et al. 1990). The reasons for the characteristic muscle damage, even at baseline, remain to be clearly elucidated. The mechanical stress imposed by high muscle glycogen stores, or the downregulation of $\mathrm{Na}^{+}-\mathrm{K}^{+}$ pumps in patients' muscles (with these pumps being responsible for maintaining cellular volume and integrity), are candidates to contribute, together with increase oxidative stress, to structural muscle fiber fragility and membrane disruption, leading to the efflux of intracytoplasmic proteins such as CK into the bloodstream (Santalla et al. 2014a, b). Most studies on healthy people show that CK levels correlate well with measures of muscle mass (Noori et al. 2011; Patel et al. 2013), and low CK levels are associated with low values of muscle mass (Oterdoom et al. 2009). On the other hand, the low values of LM mass recently reported by us in adult (mean age $33 \pm$ 15 years) McArdle patients could be explained by the fact that the muscle damage-induced stimulus for muscle tissue hypertrophy does not compensate for the detrimental effects of lack of sports practice over decades.

Our study is not without limitations. We did not report nutritional data, and dietary factors, particularly calcium and vitamin D intake, are influential for bone mass acquisition (Smith et al. 2017). However, such dietary factors might not be determinant in childhood. For instance, vitamin D and calcium supplementation does not appear to affect bone mass in another pediatric condition, acute lymphoblastic leukemia
(Demirsoy et al. 2017). Secondly, it would have also been useful to objectively assess physical activity levels in our participants; because physical activity and muscular development are major determinants of bone mass acquisition and low physical activity is associated with a reduced bone mass (Vicente-Rodriguez et al. 2005). Nonetheless, this is the first study to objectively assess body composition in McArdle children and adolescents using the gold standard DXA method, and we studied a representative sample of children. This is important when considering that only $5 \%$ of Spanish McArdle patients are correctly diagnosed during the first decade of life (Rodríguez-Gómez et al. 2018), and there is insufficient awareness and monitoring of this disease, especially among pediatricians (Alfredo Santalla et al. 2017).

In conclusion, we have identified a novel finding, nonosteogenic muscle hypertrophy, in children with McArdle disease. Thus, the recently identified problem of poor bone health in adult patients appears to start during childhood (RodríguezGómez et al. 2018). Future research might determine if specific dietary interventions (e.g., calcium supplement intake) and/or structured exercise can attenuate this problem in this period of life, and which exercise modality would be more appropriate for children with this characteristic to maximize bone mass accretion.

Acknowledgments This study was funded by the Cátedra Real Madrid Universidad Europea de Madrid (P2016/RM25), Fondo de Investigaciones Sanitarias (A.L., PI15/00558; G.N.G, PI15/01756 and CP14/00032, J.A. PI14/00903), AFM Telethon Trampoline Grant \#21108, the Biomedical Research Networking Center on Frailty and Healthy Aging (CIBERFES) and FEDER funds from the European Union (CB16/10/00477). Irene Rodríguez Gómez has received a $\mathrm{PhD}$ grant from the Universidad de Castilla-La Mancha "Contratos predoctorales para la formación de personal investigador en el marco del Plan Propio de I + D +i, cofinanciados por el Fondo Social Europeo" (2014/10340). 


\section{Compliance with ethical standards}

Conflict of interest None.

\section{References}

Bachrach LK (2014) Diagnosis and treatment of pediatric osteoporosis. Curr Opin Endocrinol Diabet Obes 21(6):454-460

Bertoldo F, Zappini F, Brigo M et al (2015) Prevalence of asymptomatic vertebral fractures in late-onset Pompe disease. J Clin Endocrinol Metab 100(2):401-406. https://doi.org/10.1210/jc.2014-2763

Demirsoy U, Sarper N, Gelen SA, Zengin E, Kum T, Demir H (2017) The association of oral vitamin D and calcium supplementation with bone mineral density in pediatric acute lymphoblastic leukemia patients. J Pediatr Hematol Oncol 39(4):287-292

Evans WJ, Cannon JG (1991) 3 the metabolic effects of exercise-induced muscle damage. Exerc Sport Sci Rev 19(1):99-126

Garcia-Benitez S, Fleck SJ, Naclerio F, Martin MA, Lucia A (2013) Resistance (weight lifting) training in an adolescent with McArdle disease. J Child Neurol 28(6):805-808. https://doi.org/10.1177/ 0883073812451328

Golden NH, Abrams SA (2014) Optimizing bone health in children and adolescents. Pediatrics 134(4):e1229-e1243

Komulainen J, Kalliokoski R, Koskinen S, Drost M, Kuipers H, Hesselink M (2000) Controlled lengthening or shortening contraction-induced damage is followed by fiber hypertrophy in rat skeletal muscle. Int J Sports Med 21(02):107-112

Lucia A, Ruiz JR, Santalla A et al (2012) Genotypic and phenotypic features of McArdle disease: insights from the Spanish national registry. J Neurol Neurosurg Psychiatry 83(3):322-328. https://doi. org/10.1136/jnnp-2011-301593

Marrani E, Giani T, Simonini G, Cimaz R (2017) Pediatric osteoporosis: diagnosis and treatment considerations. Drugs 77(6):679-695. https://doi.org/10.1007/s40265-017-0715-3

Melis D, Rossi A, Pivonello R et al (2016) Reduced bone mineral density in glycogen storage disease type III: evidence for a possible connection between metabolic imbalance and bone homeostasis. Bone 86: 79-85. https://doi.org/10.1016/j.bone.2016.02.012

Mitchell JA, Chesi A, Elci O et al (2015) Genetics of bone mass in childhood and adolescence: effects of sex and maturation interactions. J Bone Miner Res 30(9):1676-1683

Nogales-Gadea G, Santalla A, Ballester-Lopez A et al (2016) Exercise and preexercise nutrition as treatment for McArdle disease. Med Sci Sports Exerc 48(4):673-679. https://doi.org/10.1249/MSS. 0000000000000812

Noori N, Kovesdy CP, Bross R et al (2011) Novel equations to estimate lean body mass in maintenance hemodialysis patients. Am J Kidney Dis 57(1):130-139

Oterdoom LH, Gansevoort RT, Schouten JP, de Jong PE, Gans RO, Bakker SJ (2009) Urinary creatinine excretion, an indirect measure of muscle mass, is an independent predictor of cardiovascular disease and mortality in the general population. Atherosclerosis 207(2): 534-540

Patel SS, Molnar MZ, Tayek JA et al (2013) Serum creatinine as a marker of muscle mass in chronic kidney disease: results of a cross-sectional study and review of literature. J Cachexia Sarcopenia Muscle 4(1): 19-29

Perez M, Mate-Munoz JL, Foster C et al (2007) Exercise capacity in a child with McArdle disease. J Child Neurol 22(7):880-882. https:// doi.org/10.1177/0883073807304206

Rake JP, Visser G, Huismans D et al (2003) Bone mineral density in children, adolescents and adults with glycogen storage disease type Ia: a cross-sectional and longitudinal study. J Inherit Metab Dis 26(4):371-384

Rauch F, Bailey DA, Baxter-Jones A, Mirwald R, Faulkner R (2004) The 'muscle-bone unit'during the pubertal growth spurt. Bone 34(5): $771-775$

Rizzoli R, Bianchi ML, Garabédian M, McKay HA, Moreno LA (2010) Maximizing bone mineral mass gain during growth for the prevention of fractures in the adolescents and the elderly. Bone 46(2): 294-305

Rodríguez-Gómez I, Santalla A, Diez-Bermejo J et al (2018) A new condition in McArdle disease: poor bone health - benefits of an active lifestyle. Med Sci Sports Exerc 50(1):3-10. https://doi.org/ 10.1249/MSS.0000000000001414

Santalla A, Munguía-Izquierdo D, Brea-Alejo L et al (2014a) Feasibility of resistance training in adult McArdle patients: clinical outcomes and muscle strength and mass benefits. Front Aging Neurosci 6:334

Santalla A, Nogales-Gadea G, Ortenblad N et al (2014b) McArdle disease: a unique study model in sports medicine. Sports Med 44(11): 1531-1544. https://doi.org/10.1007/s40279-014-0223-5

Santalla A, Nogales-Gadea G, Encinar AB et al (2017) Genotypic and phenotypic features of all Spanish patients with McArdle disease: a 2016 update. BMC Genomics 18(8):819

Scalco RS, Morrow JM, Booth S, Chatfield S, Godfrey R, Quinlivan R (2017) Misdiagnosis is an important factor for diagnostic delay in McArdle disease. Neuromuscular Disord 27(9):852-855. https://doi. org/10.1016/j.nmd.2017.04.013

Schoenau E (2005) From mechanostat theory to development of the" functional muscle-bone-unit". J Musculoskelet Nueronal Interact 5(3):232

Schoenau E, Frost H (2002) The" muscle-bone unit" in children and adolescents. Calcif Tissue Int 70(5):405-407

Schonau E, Schwahn B, Rauch F (2002) The muscle-bone relationship: methods and management - perspectives in glycogen storage disease. Eur J Pediatr 161(Suppl 1):S50-S52. https://doi.org/10.1007/ s00431-002-1003-z

Schwahn B, Rauch F, Wendel U, Schonau E (2002) Low bone mass in glycogen storage disease type 1 is associated with reduced muscle force and poor metabolic control. J Pediatr 141(3):350-356. https:// doi.org/10.1067/mpd.2002.126456

Smith TJ, Tripkovic L, Lanham-New SA, Hart KH (2017) Vitamin D in adolescence: evidence-based dietary requirements and implications for public health policy. Proc Nutri Soc 1-10

Vicente-Rodríguez G (2006) How does exercise affect bone development during growth? Sports Med 36(7):561-569

Vicente-Rodriguez G, Ara I, Pérez-Gómez J, Dorado C, Calbet JA (2005) Muscular development and physical activity as major determinants of femoral bone mass acquisition during growth. Br J Sports Med 39(9):611-616

Wernig A, Irintchev A, Weisshaupt P (1990) Muscle injury, crosssectional area and fibre type distribution in mouse soleus after intermittent wheel-running. J Physiol 428(1):639-652 